\title{
Efecto de un programa de Hatha Yoga en pacientes con enfermedad de Alzheimer $(E A)^{1}$
}

\author{
María Clara Rodríguez Salazar*, Alba Lucía Meneses Báez, Eliana Alexey Quintero Gallego y Lina María Rodríguez Granada. \\ Universidad El Bosque
}

Recibido, noviembre 27/2015

Concepto de evaluación, junio 22/2016

Aceptado, octubre 21/2016
Referencia: Rodríguez Salazar, M.C., Meneses Báez, A.L., Quintero Gallego, E.A. \& Rodríguez Granada, L.M. (2017). Efecto de un programa de hatha yoga en pacientes con Alzheimer. Acta Colombiana de Psicología, 20(1), 123-138. DOI: 10.14718/ACP.2017.20.1.7

\section{Resumen}

\begin{abstract}
En este estudio se evaluó el efecto de un programa de hatha yoga en 20 hombres y 41 mujeres con promedio de edad de 76.9 años (DE = 11.7), con diagnóstico de Enfermedad de Alzheimer (EA) que presentaban deterioro cognitivo global leve (36 \%) y moderado (64 \%). El diseño fue cuasi experimental de medidas repetidas (pre, post y seguimiento). Se utilizó la escala de Calidad de Vida para pacientes con Enfermedad de Alzheimer (ADRQL), el cuestionario de Calidad de Vida (CV), la escala de depresión geriátrica de Yesavage, el Minimental State Examination (MMSE), dos subpruebas de la escala de inteligencia para adultos del WAIS-III (retención de dígitos y claves), la prueba de trazado Trail Making Test (TMT-A), la prueba de Lawton y Brody, y la escala de Barthel (actividades básicas y funcionales). El programa contó con 32 sesiones de hatha yoga de una hora, realizadas dos veces por semana. El análisis de datos se hizo con 35 pacientes que permanecieron hasta la fase de seguimiento. Como resultados se obtuvo que entre pre-tratamiento y pos-tratamiento las variables mostraron una tasa incremental de mejoramiento en calidad de vida y atención; pero dichas mejorías no se mantuvieron seis meses más tarde; el ANOVA de medidas repetidas no mostró cambios significativos en ninguna variable; y el análisis cualitativo de ocho grupos focales con pacientes y cuidadores dio cuenta de la percepción cambios positivos en los pacientes con respecto a su estado de ánimo, atención, memoria episódica y procedimental. Se necesitan más estudios con mayor número de pacientes y sesiones de yoga por semana, además de un grupo control, para mejorar la validez de los resultados.

Palabras clave: Hatha yoga, enfermedad de Alzheimer, cognición, calidad de vida, depresión, funcionalidad.
\end{abstract}

\section{Hatha Yoga effects on Alzheimer patients (AP)}

\begin{abstract}
This study assessed the effect of a Hatha yoga program in 20 men and 41 women diagnosed with Alzheimer's Disease (AD), who showed mild (36\%) and moderate (64 \%) global cognitive decline and whose average age was 76,9 years old $(\mathrm{SD}=11,7)$. The design was quasi-experimental of repeated measures (pre, post and follow-up). The instruments applied were: Alzheimer's Disease Related Quality of Life Scale (ADRQL); Questionnaire of Quality of Life (QQL), Geriatric Depression Scale (YESAVAGE); Mini Mental State Examination (MMSE); memory and processing speed sub-scales of WAIS III test, Trail Making Test (TMT-A); Lawton and Brody Test; and Barthel Test. The yoga program consisted of 32 one hour sessions twice a week Data analysis was conducted using 35 patients who participated throughout the whole study. The post-treatment mean showed a positive improvement tendency in quality of life and attention. However, during the follow-up, these variables mean value was equal to, or even lower, than the one established in the pre-treatment. The repeated measures ANOVA results showed that no significant change occurred between measurements. The content analysis of eight focus groups suggests that, from the perspective of patients and caregivers, positive changes were observed in patients' mood, attention, episodic and procedural memory, and functionality. More studies are necessary with a higher number of patients and sessions per week, as well as a control group, whose function would be to increase validity of the results obtained.

Key words: Hatha yoga, Alzheimer's disease, attention, quality of life, depression, functionality.
\end{abstract}

\footnotetext{
* Directora de la Especialización Psicología del Deporte y el Ejercicio, Facultad de Psicología, Universidad El Bosque, Calle 145 \#12-45, teléfono 571-6489030. Bogotá, Colombia, rodriguezmariac@unbosque.edu.co

${ }^{1}$ Proyecto financiado por Colciencias: convocatoria 545 de 2011, código 130854531719.
} 


\title{
Efeito de um Programa de Hatha Yoga em Pacientes com Alzheimer
}

\author{
Resumo
}

\begin{abstract}
Neste estudo, avaliou-se o efeito de um programa de hatha yoga em 20 homens e 41 mulheres com idade média de 76.9 años (DE = 11.7), com diagnóstico de Doença de Alzheimer (DA) que apresentavam deterioração cognitiva global leve (36 \%) e moderada (64\%). O desenho foi quase-experimental de medidas repetitivas (pré, pós e seguimento). Utilizaram-se a escala de qualidade de vida para pacientes com Alzheimer (ADRQL, em inglês), o questionário de qualidade de vida (QV), a escala de depressão geriátrica de Yesavage, o Minimental State Examination (MMSE), dois subtestes da escala de inteligência para adultos do WAIS-III (retenção de dígitos e códigos), o Trail Making Test (TMT-A), o teste de Lawton e Brody, e a escala de Barthel (atividades básicas e funcionais). O programa contou com 32 sessões de hatha yoga de uma hora, realizadas duas vezes por semana. A análise de dados foi feita com 35 pacientes que permaneceram até a fase de seguimento. Como resultados, obteve-se que, entre pré-tratamento e pós-tratamento, as variáveis mostraram uma taxa incremental de melhoramento em QV e atenção; contudo, essas melhorias não se mantiveram seis meses depois; o ANOVA de medidas repetidas não mostrou mudanças significativas em nenhuma variável; a análise qualitativa de oito grupos focais com pacientes e cuidadores indicou que estes perceberam mudanças positivas nos pacientes com relação ao seu estado de humor, atenção, memória episódica e procedimental. São necessários mais estudos com maior número de pacientes e sessões de yoga por semana, além de um grupo de controle para melhorar a validade dos resultados.

Palavras-chave: hatha yoga, doença de Alzheimer, cognição, , qualidade de vida, depressão, funcionalidade.
\end{abstract}

\section{INTRODUCCIÓN}

Según la Organización Mundial de la Salud (2012), la demencia es definida como una enfermedad cerebral crónica y progresiva que altera la memoria, el pensamiento la orientación, el lenguaje, la capacidad de aprendizaje y la toma de decisiones; así como el control emocional, la motivación y el comportamiento social. Los tipos más comunes de demencia son la demencia tipo Alzheimer (EA), la vascular, la demencia de cuerpos de Lewy y la frontotemporal; siendo frecuentes las patologías mixtas (Neuropathology Group of the Medical Research Council Cognitive Function and Aging Study, 2001; Niedermeyer \& Ghigo, 2011).

En el mundo hay unos 47.5 millones de personas que padecen demencia, cada año se esperan 7.7 millones de casos nuevos (OMS, 2012) y, debido al aumento de la expectativa de vida y a la mayor precisión en el diagnóstico, se estima que para el año 2030 habrán aproximadamente 70 millones de casos de EA (Ferri et al., 2005).

Con respecto al costo anual mundial de la demencia, para el año 2005 se calculó en 215 mil millones de euros, y se estimó que el gasto anual de un paciente bajo medicación era de 1825 dólares, y de 15 dólares por hora; sin embargo, el diagnóstico y el tratamiento desde estadios tempranos de la EA pueden representar un ahorro importante para el sistema de salud (Hogervorst, Ribeiro, Molyneux, Budge, \& Smith, 2002; Mittelman, Haley, Clay \& Rith, 2006; Skoog et al., 1996).

En Latinoamérica, la prevalencia de la EA es de $6.7 \%$ en Perú, $8 \%$ en Venezuela, $11.5 \%$ en Argentina y $5.3 \%$ en Brasil (Custodio, Gutiérrez \& García, 2007; Molero, Pino-Ramírez \& Maestre, 2007; Pages-Larraya, Grasso \& Mari, 2004).
Mientras que en Colombia, en el 2002, dicha prevalencia fue de $1.8 \%$ en pacientes entre 65 y 74 años, y de $3.4 \%$ en población mayor de 75 años; las regiones con mayor prevalencia fueron la suroccidental (2.2\%) y la oriental (1.9\%) (Pradilla, et al., 2002; Rosselli et al., 2000). En la Clínica de Memoria de la Universidad Javeriana en Bogotá se reportó que durante el período 1997-2009, de 2018 pacientes diagnosticados, el $49.56 \%$ tenía EA, de los cuales el $28.4 \%$ eran mujeres y el $71.6 \%$ hombres (Ruiz et al., 2009).

Esta enfermedad se caracteriza por un curso insidioso y por la triada de síntomas de amnesia, anomia y apraxia; se desarrolla por una combinación de factores (ambientales, genéticos y de estilo de vida); y se puede presentar en cualquier momento de la vida, aunque es más común en adultos mayores, sin ser parte del proceso normal de envejecimiento (OMS, 2012).

Aunque hasta el momento actual la EA no tiene cura, los tratamientos tanto farmacológicos como no farmacológicos están orientados a disminuir la sintomatología afectiva, mantener estable el cuadro clínico y, principalmente, favorecer la calidad de vida de la persona afectada con la enfermedad. En el tratamiento farmacológico se aborda el manejo de algunos síntomas e incluyen antidepresivos, inhibidores de la acetilcolinesterasa, antipsicóticos y sedantes, entre otros. Mientras que en los programas de intervención no farmacológicos la modalidad del tratamiento depende del grado de deterioro del paciente, pues en las primeras fases se busca estimular o mantener procesos cognitivos y funcionales, y en fases más avanzadas el objetivo es compensar las dificultades a través de ayudas externas (OMS, 2012).

Últimamente, en los programas no farmacológicos se le ha dado una mayor importancia a la actividad física, que 
tiene como propósito mantener la movilidad, el equilibrio, reducir el riesgo de caídas y mejorar el estado de ánimo. Esta tendencia se basa en los resultados de estudios epidemiológicos que señalan la relación existente entre actividad física y funcionamiento cognitivo (OMS, 2012).

Además, se ha encontrado que implicar a pacientes con EA en rutinas de actividades diarias que incluyan actividad física mejora su fuerza muscular, resistencia cardiorrespiratoria, equilibrio, flexibilidad, densidad ósea, calidad del sueño, funcionalidad e interacción social; además de que disminuye las alteraciones de comportamiento y emocionales (Arlene, Marieke \& David, 2010; Chen, et al., 2008; Dayanim, 2009; Graff-Radford, 2011; Gudlaugsson, et al., 2013; Liu \& Latham, 2009; Oken et al., 2006; Radak, et al., 2010; Ramos-Jiménez, et al., 2009; U.S. Departament of Health and Human Services, 2008; Yu \& Kolanowski, 2009). El US Department of Health and Human Services (2008) recomienda que los programas de actividad física para personas diagnosticadas con EA deben diseñarse incluyendo actividades aeróbicas de una duración mínima de 150 minutos por semana, además de incluir actividades de fuerza al menos dos veces por semana. Sin embargo, los mejores resultados se han obtenido al combinar la actividad física con tareas cognoscitivas (Winchester et al., 2013).

Los efectos del ejercicio físico en pacientes con EA se explican, primero, porque produce cambios metabólicos y neurológicos asociados a la disminución en la producción anómala de la proteína $\beta$-amiloide en la corteza frontal y el hipocampo; incrementa la circulación sanguínea en los lóbulos frontales, parietales y temporales, y propicia el crecimiento de capilares en la corteza primaria motora; además de que promueve la regulación del óxido nítrico que estimula los factores asociados al crecimiento nervioso, la producción y el funcionamiento de neurotransmisores; y aumenta los niveles de calcio, que a su vez estimula la síntesis de la dopamina. En segundo lugar, la actividad física incrementa la producción de endorfinas, las cuales contribuyen a disminuir la depresión y a favorecer sentimientos de bienestar; además de que, al igual que en las personas sanas, genera mayor producción de endorfinas, mayor funcionalidad y la mejora de cualidades físicas como la fuerza, la flexibilidad y el equilibrio (Pérez \& Cancela-Carral, 2008).

Teniendo esto en cuenta, Coehlo et al. (2012) realizaron un estudio con 27 personas con EA en el que aplicaron un programa de 16 semanas, de complejidad progresiva, que incluyó ejercicios aeróbicos, de fuerza, flexibilidad, equilibrio, agilidad y actividades cognoscitivas; y encontraron diferencias significativas a favor del grupo experimental en sus funciones ejecutivas y la velocidad de procesamiento de información, además de un menor deterioro.
Por su parte, De Andrade et al. (2013) llevaron a cabo un estudio con el objetivo de mejorar la condición física y las funciones ejecutivas de 30 pacientes con EA, todos con intervención farmacológica. Para ello dividieron el grupo en dos, y en el experimental aumentaron el nivel de dificultad de la actividad física cada cuatro semanas hasta un máximo de 65-75\% de la frecuencia cardiaca máxima. Los resultados evidenciaron mayor funcionalidad y desempeño en funciones ejecutivas en los pacientes de este grupo, así como mejores puntajes en la velocidad de procesamiento, sin embargo, las diferencias no fueron estadísticamente significativas.

Además, Winchester et al. (2013) condujeron un estudio durante un año con 104 pacientes con EA mayores de 60 años en el que aplicaron un programa de actividad física compuesto por diversas actividades, como bolos, ciclismo, caminatas, natación, yoga, entrenamiento de fuerza y excursionismo. El cuidador debía registrar la cantidad de tiempo que el paciente le dedicaba a estas actividades cada semana y la intensidad con que las realizaba. Como resultados se encontró que las personas que caminaban de manera constante obtuvieron puntajes más estables en el MMSE al final de los 12 meses; y se evidenció un estado emocional más positivo en quienes realizaban durante mayor tiempo la actividad física, aunque no se encontraron diferencias en el comportamiento, en la capacidad funcional ni en los síntomas depresivos.

Específicamente, se han hecho estudios que tienen en cuenta al hatha yoga como actividad física y que utilizan intervenciones exclusivas o en combinación con tratamientos farmacológicos tradicionales, en casos de cáncer (Cramer, et al., 2012; Smith \& Pukall, 2009), síntomas de menopausia (Cramer, Lauche, Langhorst \& Dobos, 2012), dolores crónicos (Li \& Goldsmith, 2012), ansiedad y esquizofrenia (Cramer, Lauche, \& Dobos, 2014; Vancampfort, et al., 2012), entre otras patologías. Estos programas se basan en posturas corporales (asanas) y movimientos lentos y conscientes que se acompañan con técnicas de respiración (pranayama) y ejercicios de meditación dirigidos hacia la focalización de la atención (dyana); que cuentan con numerosas ventajas y consecuencias favorables para los pacientes.

Por ejemplo, Sengupta (2012) señala que el yoga disminuye el arousal, la frecuencia cardiaca, la presión sanguínea y facilita la respiración; además de que aumenta la tolerancia al dolor y los niveles de melatonina en la sangre; y mejora la variabilidad cardiaca, que es un indicador de adecuada respuesta al estrés (Madanmohan, Rai, Balavittal \& Thombre citados por Sengupta, 2012; Smith, Hancock, Blake-Mortimer \& Eckert, 2007; Tooley, Armstrong, Norman \& Sali, 2000). 
Incluso algunos estudios, como el de Kjaer et al. (2002), han demostrado que la práctica de hatha yoga puede aumentar la liberación de dopamina endógena en el estriado ventral y en los niveles de GABA talámicos; asimismo, está asociada con el aumento de serotonina en el plasma en pacientes con depresión (Devi, Chansauria \& Udupa citados por Cramer et al., 2014). También hay evidencia de que esta práctica regula el eje hipotálamo-hipófisis-suprarrenal, encargado de las respuestas de estrés (Streeter, Gerbarg, Saper, Ciraulo \& Brown, 2012) y disminuye los niveles de cortisol plasmático (Jarcho, Slavich, Tylova-Stein, Wolkowitz, \& Burke, 2013; Gartside, Leitch, McQuade \& Swarbrick, 2003; Vadiraja et al., 2009). Sin embargo, a pesar de los avances mencionados, estos estudios no han sido concluyentes debido a dificultades metodológicas (Li \& Goldsmith, 2012).

Por otra parte, Khoo, Van Schaik y McKeena (2014) realizaron un estudio que tuvo como objetivo evaluar el ejercicio holístico en 15 adultos mayores con demencia. Las sesiones del Happy Antics Programe se realizaron una vez por semana en sesiones de 45 minutos durante seis semanas (con un $70 \%$ de asistencia); sesiones en las que se incluían ejercicios coreografiados con música de tai-chi, meditación, qigong, baile y mindfulness; y ejercicios cognitivos basados en la presentación de imágenes y discusiones con respecto a ellas. Al finalizar la última sesión, mediante una entrevista semiestructurada, se encontró que todos los participantes disfrutaron del programa, lo percibieron como una oportunidad para socializar y adquirir nuevos aprendizajes, y, además, algunos reportaron disminución de dolores y relajación después de las sesiones. Adicionalmente, cabe mencionar que seis de las participantes podían anticipar los movimientos asociándolos con la música y tres podían recordar secuencias de movimientos completas.

Dichos resultados son consistentes con los de Barnes, Mehlin, Taffe, Flores y Chesney (2013), que evaluaron el programa PLIÉ (Preventing Loss of Independence through Exercise), el cual, aunque no incluye ejercicios de estimulación cognoscitiva, se basa en la terapia física y ocupacional, el yoga y la danza para emplear seis métodos que buscan mantener a los pacientes activos: (a) repetición con variaciones, (b) movimientos progresivos y funcionales, (c) instrucciones paso a paso, (d) orientación de metas, (e) cuidado físico, mindfulness y respiración, y (f) interacción social.

Por su parte, Fan y Chen (2011) evaluaron los efectos de un programa de silver-yoga (combinación de hatha yoga con principios de gerontología) sobre la salud física y mental de 68personas mayores con demencia. La intervención consistió en tres sesiones semanales de 55 minutos, durante 12 semanas, que incluían calentamiento, hatha yoga, y relajación; y en las que participaban dos instructores, uno como modelo y otro como apoyo. Se encontraron diferencias significativas entre las medidas pre y post del grupo experimental, y entre los grupos control y experimental en variables físicas, como presión sanguínea, frecuencia respiratoria, condición cardiopulmonar, flexibilidad, fuerza de agarre, resistencia muscular, movilidad articular y equilibrio. Además, se encontró disminución de los problemas de comportamiento y depresión, e incremento en las interacciones entre los participantes. Estos resultados son coherentes con los de otros estudios (Arlene et al., 2010; Chen et al., 2008; McCaffrey, Ruknui, Hatthakit, \& Kasetsomboon, 2014; Oken et al., 2006; Ramos-Jiménez et al., 2009).

También, Cramer et al. (2014) realizaron un meta-análisis para determinar el efecto del yoga en pacientes con desórdenes depresivos diferenciando efectos a corto y largo plazo (12 semanas y 12 meses respectivamente). Los resultados revelaron un efecto moderado a corto, pero no a largo plazo; se evidenció mayor efectividad en pacientes con elevados niveles de depresión que en los diagnosticados con desorden depresivo; y se encontró que los efectos del yoga eran comparables con los de tratamientos farmacológicos y terapias de grupo.

Y por último, según señalan Oken et al. (2006), existen al menos dos mecanismos potenciales por los cuales la práctica tanto del hatha yoga como del ejercicio en general podrían optimizar la habilidad cognitiva: ambas actividades mejoran el estado del ánimo y reducen el estrés (asociados con la disminución cognitiva) y, teniendo en cuenta que la práctica del yoga hace énfasis en la conciencia sobre el cuerpo y el hecho de que concentrarse en la respiración o en músculos específicos podría estimular la atención, este proceso atencional se vería fortalecido, proceso fundamental para la realización de diferentes tareas en la vida cotidiana y necesario para el aprendizaje, la memoria y el lenguaje.

Esta revisión de literatura evidencia entonces un amplio interés científico sobre intervenciones basadas en actividad física y específicamente sobre el hatha yoga en pacientes con demencia tipo Alzhéimer, interés asociado al crecimiento de la población adulta mayor a nivel mundial; sin embargo, aunque las cifras de personas diagnosticadas con demencia crecen año tras año, en Colombia no hay estudios acerca del tema. Teniendo en cuenta lo anterior, el presente trabajo tiene como objetivo determinar el efecto de un programa de hatha yoga sobre variables psicológicas (calidad de vida, depresión, atención, memoria y funcionamiento cognitivo global) y funcionales (independencia para la realización de actividades básicas e instrumentales de la vida diaria), en pacientes diagnosticados con demencia tipo Alzheimer en la ciudad de Bogotá. 


\section{MÉTODO}

\section{Tipo de estudio}

El presente es un estudio cuasi-experimental de medidas repetidas, sin grupo control.

\section{Participantes}

La muestra de participantes se seleccionó por conveniencia y estuvo conformada por 61 pacientes y sus cuidadores, quienes aceptaron participar en el estudio de forma voluntaria y firmaron el consentimiento informado. En los criterios de inclusión se tuvo en cuenta que los participantes tuvieran diagnóstico de enfermedad de Alzheimer por consenso de equipo interdisciplinario (conformado por neurólogo, psiquiatra, geriatra, neuropsicólogo, psicólogo y terapeuta ocupacional), nivel de deterioro entre leve y moderado según la Global Deterioration Scale (GDS), que no tuvieran problemas físicos o sensoriales que les impidieran participar en el programa de yoga, y que contaran con afiliación a una Empresa Promotora de Salud (EPS) y con un cuidador que los acompañara durante el proceso.

Ninguno de los pacientes reportó estar vinculado a clases de yoga al inicio de su participación en este estudio, el $48 \%$ de nunca había asistido a una clase de yoga y el 22 \% había tomado clases durante por lo menos un año; solo un paciente manifestó haber tomado clases por siete años. Según los cuidadores, al inicio del programa el 72 \% de los pacientes presentaba disminución auditiva, el $60 \%$ utilizaba gafas para leer y el 23 \% tenía alguna patología ocular (en su mayoría cataratas). Estas alteraciones sensoriales no afectaron la ejecución de las pruebas ni tampoco su participación en el programa. Además, el 18 \% reportó haber sufrido golpes en la cabeza previos al inicio de la enfermedad, sin evidencia de secuelas neurológicas; $y$ todos los pacientes reportaron contar con una adecuada red de apoyo familiar, compuesta en la mayoría de los casos por sus cónyuges, hijos y otros familiares (87 \%) (véase Tabla 1).

\section{Instrumentos}

Se utilizaron los siguientes instrumentos:

Cuestionario de Calidad de Vida. Construido por las autoras de este estudio para medir la calidad de vida en personas con demencia; es una escala que se aplica al cuidador y consta de 25 ítems tipo likert ( 0 = nunca y 4 = siempre), en donde a mayor puntaje corresponde mejor calidad de vida. Este instrumento evalúa funcionalidad, actividad física, afectividad, relaciones interpersonales y aspectos cognoscitivos. Con base en los datos de 59 pacientes (40 mujeres y 19 hombres) con diagnóstico de Alzheimer, con promedio de edad de 75.4 y DE = 11.2, y con GDS entre leve y moderado, se estimó el Alpha de Cronbach para la escala CV total $(r=.81)$ y para cada una de las sub escalas: funcional, físico, afectivo, interpersonal y cognitivo ( $r=$ $.62 ; r=.58 ; r=.88 ; \mathrm{r}=.51 ; \mathrm{y} r=.68$, respectivamente). El coeficiente de correlación de Pearson entre las puntuaciones totales del cuestionario de CV y ADRQL fue de $r=.714$ $(\mathrm{p}<.001)$, resultado que sugiere que las pruebas miden un constructo similar: la calidad de vida.

Escala de Calidad de Vida en la Enfermedad de Alzheimer (ADRQL). Desarrollada por Hophins (2011), esta escala evalúa la calidad de vida en personas con EA, es heteroaplicada al cuidador, y consta de 47 ítems con tipo de respuesta "de acuerdo” y “en desacuerdo”, en los que se evalúan cinco sub escalas: (a) relaciones con otras personas, (b) identidad personal y de personas importantes, (c) conductas estresantes, (d) actividades cotidianas, y (e) comportamiento en el lugar de residencia. A mayor puntaje, mejor calidad de vida. Con base en los datos de 59 pacientes con diagnóstico de Alzheimer con GDS entre leve y moderado (40 mujeres y 19 hombres), con promedio de edad de 75.4 y DE = 11.2; el coeficiente de consistencia interna Alpha de Cronbach para el ADQRL total fue $r=.79$ y para cada una de las sub escalas ( $r=.61 ; r=.59 ; r=.68 ; \mathrm{r}=.45 \mathrm{y} r=.54$, respectivamente).

Escala de depresión geriátrica (Yesavage). En su versión corta, de 15 ítems, validada por Martínez de la Iglesia, et al. (2002) para la población española mayor de 65 años, evalúa síntomas depresivos. El examinado debe responder “Sí” (puntuación de 1) o "No" (puntuación de 0) a 15 afirmaciones leídas por el examinador. En los resultados se considera que los puntajes de 0 a 5 son "normales", de 6 a 10 representan "depresión moderada”, y de 11 a 15, “depresión severa”. Esta validación reporta índices de confiabilidad altos: intraobservador $(r=.95)$, interobservador ( $r=.65)$ y de consistencia interna $(r=.99)$.

Subtest: Dígito-Símbolo. Hace parte de la Escala de Inteligencia de Wechsler para Adultos (WAIS-III) (Wechsler, 1997) y evalúa la capacidad para el sostenimiento de la atención visual, la persistencia motora, la velocidad de procesamiento de información y la actividad imitativa. La tarea consiste en identificar cada número y poner debajo el símbolo correspondiente. Henao-Arboleda et al. (2010) realizaron un estudio para obtener evidencia de la confiabilidad y la validez con población adulta mayor con y sin EA. Los resultados indicaron que los coeficientes de consistencia interna son superiores a $r=.75$ para ambos tipos de muestras. También obtuvieron índices de correlación intra-clase y test-retest con diferencia de un mes y los resultados fueron superiores a $r=.75$ para ambos casos. Se observa que el desempeño en adultos mayores en esta 
Tabla 1

Características de los participantes $(n=61)$

\begin{tabular}{|c|c|c|c|}
\hline Variables & & Frecuencia & Porcentaje \\
\hline Sexo & $\begin{array}{l}\text { Hombres } \\
\text { Mujeres }\end{array}$ & $\begin{array}{l}20 \\
41\end{array}$ & $\begin{array}{l}34 \\
66\end{array}$ \\
\hline Estado Civil & $\begin{array}{l}\text { Divorciado } \\
\text { Soltero } \\
\text { Viudo } \\
\text { Casado }\end{array}$ & $\begin{array}{c}2 \\
6 \\
23 \\
30\end{array}$ & $\begin{array}{c}5 \\
10 \\
37 \\
48\end{array}$ \\
\hline Nivel escolar & $\begin{array}{l}\text { Analfabeta } \\
\text { Postgrado } \\
\text { Técnico } \\
\text { Pregrado } \\
\text { Primaria } \\
\text { Bachillerato }\end{array}$ & $\begin{array}{c}1 \\
4 \\
7 \\
12 \\
17 \\
20\end{array}$ & $\begin{array}{c}2 \\
8 \\
11 \\
19 \\
27 \\
32\end{array}$ \\
\hline $\begin{array}{l}\text { Ocupación antes de la } \\
\text { enfermedad }\end{array}$ & $\begin{array}{l}\text { Área de salud } \\
\text { Administrativo } \\
\text { Ingeniero } \\
\text { Técnico } \\
\text { Otros } \\
\text { Oficios en el hogar }\end{array}$ & $\begin{array}{c}2 \\
4 \\
4 \\
5 \\
9 \\
32\end{array}$ & $\begin{array}{c}3 \\
7 \\
7 \\
8 \\
15 \\
55\end{array}$ \\
\hline Red de apoyo & $\begin{array}{l}\text { Hermanos y empleados } \\
\text { Cónyuge e hijos } \\
\text { Hijos y otros familiares } \\
\text { Otros familiares y empleados }\end{array}$ & $\begin{array}{c}6 \\
25 \\
28 \\
2\end{array}$ & $\begin{array}{c}9 \\
41 \\
46 \\
3\end{array}$ \\
\hline $\begin{array}{l}\text { Terapias no } \\
\text { farmacológicas }\end{array}$ & $\begin{array}{l}\text { Nivel moderado } \\
\text { Nivel leve } \\
\text { Terapia física } \\
\text { Terapia musical } \\
\text { Otras terapias } \\
\text { Terapia cognoscitiva } \\
\text { Terapia ocupacional }\end{array}$ & $\begin{array}{c}22 \\
39 \\
2 \\
2 \\
8 \\
10 \\
21\end{array}$ & $\begin{array}{c}36 \\
64 \\
3 \\
3 \\
13 \\
16 \\
39\end{array}$ \\
\hline Terapias farmacológicas & $\begin{array}{l}\text { Rivastigmina } \\
\text { Otros } \\
\text { No toman medicamentos }\end{array}$ & $\begin{array}{l}18 \\
34 \\
16\end{array}$ & $\begin{array}{l}30 \\
56 \\
25\end{array}$ \\
\hline Patologías adicionales & $\begin{array}{l}\text { Colesterol y triglicéridos alto } \\
\text { Diabetes } \\
\text { Enfermedad cardiovascular } \\
\text { Enfermedad de la tiroides } \\
\text { Enfermedad pulmonar (EPOC) } \\
\text { Hipertensión } \\
\text { Sin patologías adicionales }\end{array}$ & $\begin{array}{c}8 \\
8 \\
9 \\
12 \\
5 \\
19 \\
15\end{array}$ & $\begin{array}{c}13 \\
13 \\
15 \\
20 \\
8 \\
31 \\
24\end{array}$ \\
\hline $\begin{array}{l}\text { Consumo de alcohol, } \\
\text { tabaco y otros }\end{array}$ & $\begin{array}{l}\text { Consumo de alcohol } \\
\text { Consumo de tabaco } \\
\text { No consumo }\end{array}$ & $\begin{array}{c}4 \\
8 \\
49\end{array}$ & $\begin{array}{c}6 \\
12 \\
79\end{array}$ \\
\hline $\begin{array}{l}\text { Antecedentes } \\
\text { psiquiátricos }\end{array}$ & $\begin{array}{l}\text { Ansiedad y depresión } \\
\text { Otros trastornos psiquiátricos } \\
\text { Sin antecedentes psiquiátricos }\end{array}$ & $\begin{array}{c}15 \\
5 \\
45\end{array}$ & $\begin{array}{c}24 \\
9 \\
72\end{array}$ \\
\hline $\begin{array}{l}\text { Antecedentes de } \\
\text { demencia }\end{array}$ & $\begin{array}{l}\text { Abuela } \\
\text { Tíos } \\
\text { Padres } \\
\text { Hermanos }\end{array}$ & $\begin{array}{c}3 \\
3 \\
6 \\
15\end{array}$ & $\begin{array}{c}5 \\
5 \\
9 \\
25\end{array}$ \\
\hline $\begin{array}{l}\text { Antecedentes de otros } \\
\text { trastornos mentales }\end{array}$ & $\begin{array}{l}\text { Hermanos } \\
\text { Sin antecedentes }\end{array}$ & $\begin{array}{c}1 \\
33\end{array}$ & $\begin{array}{c}2 \\
54\end{array}$ \\
\hline
\end{tabular}

Nota. Algunos totales no suman el $100 \%$ debido a datos no reportados 
prueba está en función de la edad, el nivel educativo y el deterioro cognitivo, como es el caso de los pacientes con EA.

Subtest: Retención de dígitos en orden directo e inverso. Hace parte de la Escala de Inteligencia de Wechsler para Adultos (WAIS-III) (Wechsler, 1997) que mide atención y memoria de trabajo. Está compuesto por pares de series de tres hasta nueve dígitos en la repetición directa, y de dos a ocho dígitos en la repetición inversa. Ambas tareas exigen atención auditiva y capacidad de retención a corto plazo y memoria de trabajo. Un desempeño normal de retención de dígitos hacía adelante, según Ardila, Roselli y Puente (1994), está entre $6 \pm 1$, y la retención hacía atrás está entre 4 y 5 dígitos con diferencias que se consideran normales, de 1 a 2 dígitos; además, los individuos con edades alrededor de 70 años pueden presentar un dificultades en retención de dígitos hacía atrás; y el nivel educativo del respondiente está asociado al desempeño en esta prueba (Ardila, Ostrosky-Solis, Roselli, \& Gómez, 2000). Cabe mencionar que pacientes con EA presentan un desempeño por debajo del esperado en la tarea de retención de dígitos hacia atrás y en fases avanzadas de la enfermedad se compromete también la retención de dígitos hacia adelante (Howeison \& Lezak, 2008).

Prueba de trazado Trail Making Test Forma A (TMT A). Evalúa la atención sostenida, a través de la búsqueda y seguimiento visual de 25 dígitos. El número de secuencias correctas se obtiene en un tiempo de ejecución máximo de 120 segundos. Henao et al. (2010) realizaron un estudio para obtener evidencia de confiabilidad y validez con adultos mayores con y sin EA. Los resultados indicaron que para respuestas correctas el coeficiente de consistencia interna en personas con EA fue de $r=.60$ y el índice de correlación intra-clase test-retest con diferencia de un mes de $r=.70$ en ambos casos.

Mini-Mental State Examination (MMSE). Esta prueba se utilizó teniendo en cuenta en el estudio de Ardila et al. (2000), en donde se evidencia que al ser una escala de tamizaje y proporcionar una medida de desempeño cognitivo global, esta puede ser útil para evaluar la demencia. Específicamente, evalúa la orientación, el registro de información, la atención y el cálculo, el recuerdo, el lenguaje y la construcción. El máximo puntaje a obtener es 30, pero el punto de corte asumido fue de 26 en razón al efecto que tiene un nivel educativo bajo y un deterioro cognitivo leve sobre el desempeño (Prince, Acosta, Chiu, Scazufica \& Varghese, 2003). El MMSE con un punto de corte de 25, según diez estudios, tiene una sensibilidad de .87 y una especificidad de .92; y con un punto de corte de 24 , teniendo en cuenta quince estudios, presenta una sensibilidad de .85 y una especificidad de .90 (Creavin, et al., 2016).

Escala de Barthel. Desarrollada por Mahoney y Barthel (1965), se aplica al cuidador y evalúa independencia, necesidad de ayuda o dependencia en 10 áreas de la vida diaria del paciente: alimentación, transferencias, aseo personal, uso del sanitario, baño o ducha, desplazamiento, subir y bajar escaleras, vestido, y continencia intestinal y vesical. A mayor puntaje mayor independencia. El coeficiente de consistencia interna alpha de Cronbach fue de $r=.80$ con base en los datos de 59 pacientes. Dicho índice estimado es consistente con el reportado por otros estudios (Cid-Ruzafa \& Damián-Moreno, 1997).

Escala de Lawton y Brody. Desarrollada por Lawton y Brody (1969), se aplica al cuidador y mide el nivel de independencia que un paciente tiene para realizar 14 actividades de la vida diaria de carácter instrumental. Se califica en cuatro niveles: sin dificultad, con ayuda, con dificultad y siempre con ayuda, en donde a mayor puntaje, mayor independencia. En el estudio original se estimó el alpha de Cronbach $(r=.89)$ con base en los datos reportados por los cuidadores de 59 pacientes.

\section{Procedimiento}

Para la conformación de la muestra se hicieron tres convocatorias con el Centro de Memoria y Cognición Intellectus y con la Fundación Acción Familiar Alzheimer Colombia. En total se contactaron telefónicamente a 172 familiares de pacientes con el diagnóstico. Tras la explicación del programa se inscribieron 65 personas (100\%), 61 hicieron el pre-test (94\%), 48 el pos-test (74\%), y, finalmente, 35 pacientes completaron la prueba de seguimiento (54\%).

Se ajustó el programa de hatha yoga utilizado por Quintero, Rodríguez, Guzmán, Llanos y Reyes (2011). Para ello se entrenó al instructor de yoga, experto en trabajo con adultos mayores, en habilidades para el manejo de estos pacientes, y se seleccionaron las posturas más adecuadas. Estas consistieron en movimientos de extensión y flexión de la columna vertebral que involucraban flexibilidad, equilibrio y coordinación. Se realizó un pilotaje con dos personas (un hombre y una mujer) sanas, mayores de 60 años. Con base en este se seleccionaron las posturas definitivas y se realizó un nuevo pilotaje con una persona diagnosticada con EA (mujer de 86 años, GDS 4 y con dificultades motoras leves).

Para el diseño y desarrollo del programa definitivo se tuvieron en cuenta las siguientes características: (a) instrucciones sencillas y cortas, (b) evitar nombres complejos de los segmentos del cuerpo; (c) facilitar al paciente contacto visual y auditivo con el instructor; (d) facilitar retroalimentación visual y verbal de su ejecución; (e) tocar al paciente con suavidad para corregir sus posturas y generar un aprendizaje sin errores (por el cuidador o el auxiliar de investigación), (f) que las posturas fueran las mismas durante todo el programa (para facilitar apropiación y memorización), de manera que el grado de exigencia fuera dado por el tiempo de mantenimiento de la postura 
(inicialmente más dinámicas y al final de programa más estáticas) así como la exactitud de la misma; y (g) que se entregara un video del programa para facilitar el mantenimiento de las sesiones de yoga en casa.

Cada una de las sesiones de yoga tuvo en cuenta los siguientes componentes:

Concientización. Fase inicial en la que se le pide al participante que focalice la atención en su respiración (abdominal), enfatizando en la relación con "el aquí y el ahora"; esta actividad se realiza también en otros momentos con el fin de reorientar la atención del participante al momento presente. Ejercicios de calentamiento. Con el objetivo de preparar el cuerpo para las posturas.

Posturas. Las diferentes posturas que se realizan de pie, sentado y acostado; algunas de estas se hacen de manera dinámica (repetitiva) para favorecer que el paciente flexibilice su cuerpo y paulatinamente alcance posturas fijas y mantenidas.

Relajación. Momento en el que se busca aflojar los músculos y reducir el continuo de pensamientos. Implica hacer un escáner al cuerpo y a los pensamientos para conseguir un estado de relajación y descanso.

Cierre. Se realiza al final de la sesión en postura fácil (sentado con las piernas cruzadas) con el fin de que el participante perciba los beneficios de la práctica, conecte con su cuerpo y su respiración, y observe durante unos minutos esa sensación de calma y bienestar.

El programa de yoga constó de 32 sesiones de una hora cada una, luego de la cual se ofrecía un refrigerio a pacientes y cuidadores, y se les otorgaba un subsidio para el transporte. Las sesiones se desarrollaron en un salón con adecuada iluminación y ventilación, el cual disponía de colchonetas, cobijas y almohadas con capacidad hasta para 30 personas (pacientes y cuidadores).

Para el desarrollo del programa fueron entrenadas nueve auxiliares de investigación en habilidades clínicas y de observación, las cuales, bajo la supervisión de los investigadores principales, aplicaron las pruebas y entrevistas tanto a cuidadores como a pacientes, y acompañaron y facilitaron a los participantes la apropiación de las posturas durante las sesiones.

Las evaluaciones pre tratamiento, post tratamiento y seguimiento (seis meses después de haber concluido el programa) se llevaron a cabo en el lugar de residencia de cada paciente. Una vez finalizado el programa se identificaron los pacientes que habían asistido a más del $80 \%$ de las sesiones y se les convocó junto con sus cuidadores a participar en una entrevista de grupo focal, a la que asistieron 22 participantes con sus respectivos cuidadores. Se organizaron cuatro grupos de pacientes y cuatro de cuidadores, cada uno con un promedio de cinco miembros; paraa cada uno se le solicitó la firma del consentimiento informado para la videograbación. Los registros obtenidos en los grupos focales fueron transcritos, y el objetivo de indagación fue identificar los cambios observados o percibidos en los pacientes a nivel cognitivo, afectivo, funcional y en su calidad de vida desde la participación en el programa de hatha yoga.

\section{RESULTADOS}

A continuación se presentan los resultados para cada uno de los objetivos específicos del proyecto de investigación que tenía como propósito evaluar el efecto de un programa de hatha yoga en pacientes con EA, con respecto a su calidad de vida, depresión, atención, memoria, funcionamiento cognitivo global y nivel de independencia en las actividades diarias. Para todos los análisis se utilizaron los puntajes directos de las pruebas aplicadas. Al final se presentan los resultados de la evaluación de los efectos percibidos del programa en los pacientes a través de los grupos focales en los que participaron pacientes y cuidadores.

Efecto del programa de yoga sobre la calidad de vida de pacientes con EA

Se evaluaron las diferencias de las puntuaciones obtenidas entre pre-tratamiento, post-tratamiento y seguimiento a seis meses del cuestionario de calidad de vida (CV) y de la prueba ADQRL. Los resultados del estadístico z de Kolmogorov Smirnov para las puntuaciones obtenidas con estas dos pruebas y sus dimensiones indican que los datos se ajustan a la curva normal ( $\mathrm{p}>$.05). Los estadísticos descriptivos (véase Tabla 2) indican que la media presenta tasas de crecimiento positivas similares hasta la medida de post-tratamiento, sin embargo, después de este ambas medidas muestran una tasa de crecimiento negativa similar (véase Figura 1).

Los resultados del ANOVA de medidas repetidas para la variable calidad de vida señala que no hay diferencias significativas en ninguna de las dos pruebas entre las tres mediciones $\left(\mathrm{CV} F_{2.33}=.064 ; \mathrm{p}>.05\right.$ y ADQRL $\left.F_{2.33}=.021 ; \mathrm{p}>.05\right)$.

\section{Efecto del programa de yoga sobre la depresión}

Se compararon las puntuaciones obtenidas entre las tres mediciones de la escala de depresión geriátrica Yesavage (Martínez de la Iglesia, et al., 2002). Las puntuaciones obtenidas se ajustan a la curva normal según el estadístico $\mathrm{z}$ de Kolmogorov Smirnov ( $\mathrm{p}>$.05).

Los resultados que se presentan en la Tabla 2 y en la Figura 2 indican que la media presenta una tasa de crecimiento negativa importante entre el pre-tratamiento y el post-tratamiento, y una tendencia contraria entre el post-tratamiento y el seguimiento. De cualquier manera, 
Tabla 2

Descriptivos para cada una de las variables medidas

\begin{tabular}{|c|c|c|c|c|}
\hline Cuestionario & Medición & Media & Desviación típica & $\mathrm{N}$ \\
\hline \multirow{3}{*}{ Calidad de vida } & Pre-tratamiento & 64.51 & 16.534 & 35 \\
\hline & Post-tratamiento & 69.57 & 15.422 & 35 \\
\hline & Seguimiento (6 meses) & 65.23 & 13.848 & 35 \\
\hline \multirow{3}{*}{ ADRQL } & Pre-tratamiento & 34.97 & 6.487 & 35 \\
\hline & Post-tratamiento & 36.34 & 5.173 & 35 \\
\hline & Seguimiento (6 meses) & 35.69 & 5.285 & 35 \\
\hline \multirow{3}{*}{ Yesavage } & Pre-tratamiento & 3.21 & 2.934 & 33 \\
\hline & Post-tratamiento & 2.64 & 2.356 & 33 \\
\hline & Seguimiento (6 meses) & 3.21 & 2.977 & 33 \\
\hline \multirow{3}{*}{ MMSE } & Pre-tratamiento & 19.68 & 6.475 & 34 \\
\hline & Post-tratamiento & 19.41 & 5.996 & 34 \\
\hline & Seguimiento (6 meses) & 18.68 & 6.004 & 34 \\
\hline \multirow{3}{*}{ Correctas Símbolo-Dígito } & Pre-tratamiento & 14.69 & 15.520 & 26 \\
\hline & Post-tratamiento & 15.58 & 16.221 & 26 \\
\hline & Seguimiento (6 meses) & 13.77 & 10.573 & 26 \\
\hline \multirow{3}{*}{ Correctas TMT-A } & Pre-tratamiento & 14.72 & 9.204 & 29 \\
\hline & Post-tratamiento & 16.03 & 8.781 & 29 \\
\hline & Seguimiento (6 meses) & 16.62 & 7.669 & 29 \\
\hline \multirow{3}{*}{ Retención de dígitos } & Pre-tratamiento & 8.66 & 2.947 & 32 \\
\hline & Post-tratamiento & 8.09 & 2.277 & 32 \\
\hline & Seguimiento (6 meses) & 8.38 & 2.791 & 32 \\
\hline \multirow{3}{*}{ Sin dificultad Lawton y Brody } & Pre-tratamiento & 5.97 & 3.839 & 35 \\
\hline & Post-tratamiento & 6.51 & 3.543 & 35 \\
\hline & Seguimiento (6 meses) & 5.54 & 3.156 & 35 \\
\hline \multirow{3}{*}{ BTLTotal Actividades Básicas } & Pre-tratamiento & 86.57 & 20.247 & 35 \\
\hline & Post-tratamiento & 90.43 & 13.249 & 35 \\
\hline & Seguimiento (6 meses) & 86.29 & 17.919 & 35 \\
\hline
\end{tabular}

Nota: Algunos de los totales no suman $100 \%$ debido a datos no reportados.
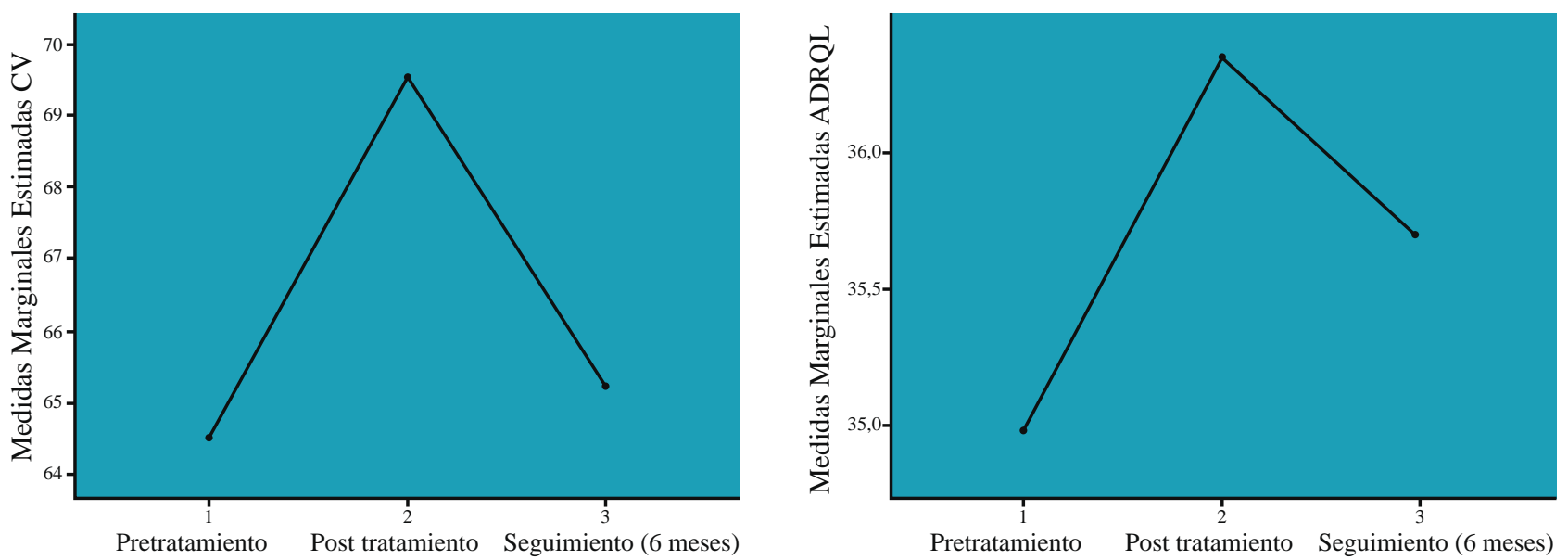

Figura 1. Media de las puntuaciones obtenidas en tres medidas en PA en los cuestionarios CV y ADQRL que miden calidad de vida $(\mathrm{n}=35)$. 
ninguna puntuación alcanza valores por fuera del rango de "normalidad" de acuerdo con la escala interpretativa del instrumento. El análisis de varianza de medidas repetidas no mostró diferencias estadísticamente significativas para esta variable $\left(F_{2.31}=.034 ; \mathrm{p}>.05\right)$ entre las tres mediciones.

Efecto del programa de yoga sobre la atención, la memoria y el funcionamiento cognitivo global

Para determinar el efecto del programa de yoga sobre la atención y la memoria de trabajo se realizó el mismo análisis a partir de las puntuaciones obtenidas en dos subtests de la escala de inteligencia Weschler (Símbolo-Dígito y Retención de dígitos); y para evaluar el funcionamiento cognitivo global se utilizó el MMSE. Se estimó el estadístico z de Kolmogorov Smirnov para establecer la normalidad de las puntuaciones obtenidas; y se encontró que los datos se ajustaron a la curva normal ( $\mathrm{p}>.05)$.

Los resultados indican que la media en el componente de atención sostenida y velocidad de procesamiento evaluada con el Símbolo-Dígito presentó una tasa de crecimiento positiva entre la medida de pre-tratamiento y post-tratamiento, mientras que a partir del post-tratamiento registra una tasa negativa de crecimiento (véanse Tabla 2 y Figura 3).

Con respecto al comportamiento de la prueba de trazado TMT-A, la media entre pre-tratamiento y post-tratamiento muestra una tasa de crecimiento que se mantiene incluso luego de la medida de post-tratamiento. Con referencia a la sub prueba de retención de dígitos, el valor de la media tiende a ser constante en las tres mediciones. En cuanto a la variable de funcionamiento cognitivo global medido por la escala de tamizaje MMSE, presenta una tasa de crecimiento negativa, la cual es más marcada una vez finalizada la intervención. El ANOVA de medidas repetidas no mostró diferencias estadísticamente significativas para ninguna de estas variables cognitivas entre las tres mediciones (Símbolo-Dígito: $F_{2.22}=.010 \mathrm{y} \mathrm{p}>.05$; Retención de dígitos: $F_{2.29}=.025 \mathrm{y} \mathrm{p}>.05$; TMT-A $F_{2.27}=.131 \mathrm{y} \mathrm{p}>.05$; MMSE: $F_{2.32}=.014$ y p $>.05$ ).

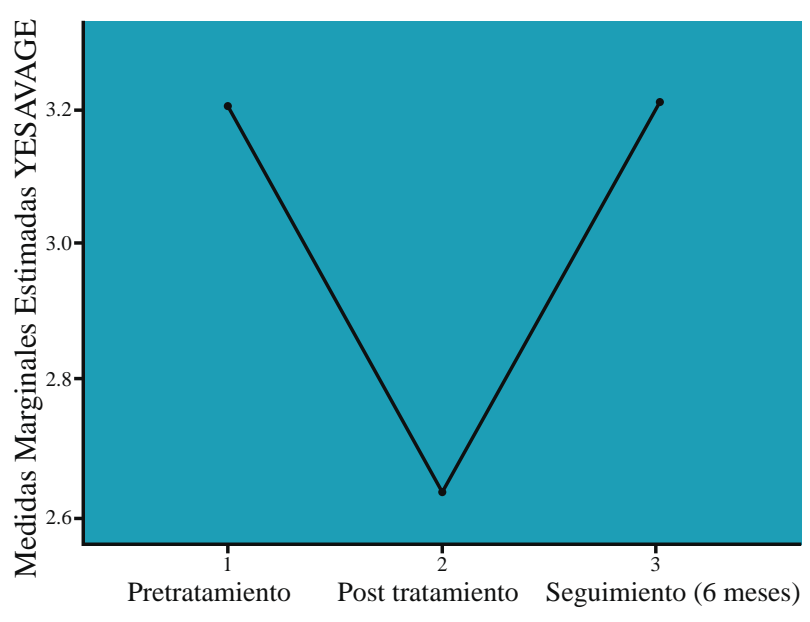

Figura 2. Media de las puntuaciones obtenidas en tres medidas en PA en la escala de depresión de Yesavage $(n=33)$.

\section{Efecto del programa de yoga sobre el nivel de independencia} en actividades de la vida diaria

Se evaluaron las diferencias entre las puntuaciones obtenidas en dos pruebas que miden actividades de la vida diaria: la Escala de Lawton y Brody, para la cual solo se tuvo en cuenta el número de actividades que el paciente realizaba de manera autónoma (sin dificultad); y la Prueba de Barthel, que mide independencia en actividades cotidianas. Se estimó el estadístico z de Kolmogorov Smirnov para establecer la normalidad de las puntuaciones en cada una de las medidas ( $\mathrm{p}>$.05).

Los resultados descriptivos que se presentan en la Tabla 2 y en la Figura 4 indican tasa de crecimiento elevada hasta el post-tratamiento, pero en la medida de seguimiento las tasas de crecimiento son negativas en ambas variables, siendo mayor en la Prueba de Lawton.

El ANOVA de medidas repetidas no mostró diferencias estadísticamente significativas para ninguna de estas dos pruebas entre las tres mediciones (Lawton: $F_{2.33}=.077 \mathrm{y}$ $\mathrm{p}>.05$; y Bartel: $F_{2.33}=.065$ y p $>.05$ ).
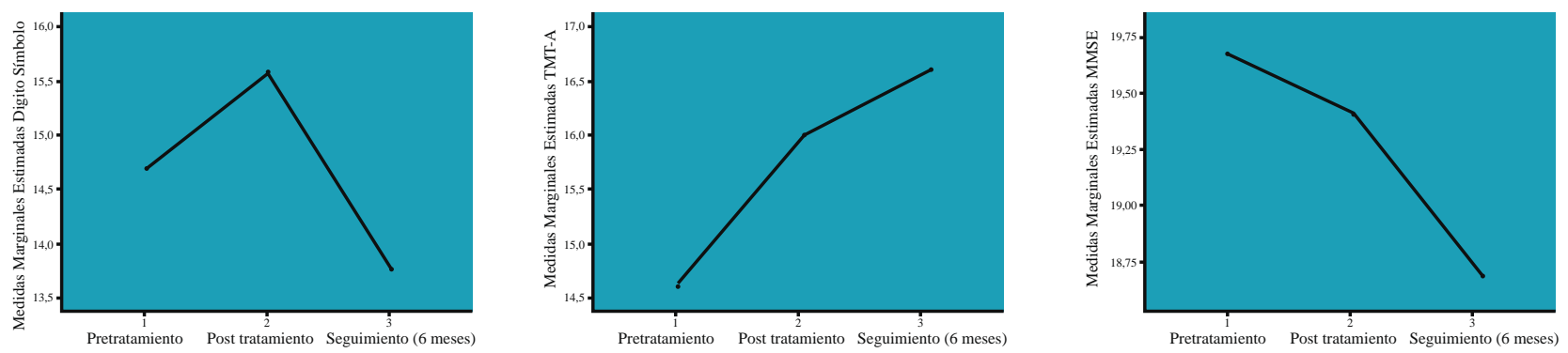

Figura 3. Media de las puntuaciones obtenidas en tres medidas en PA utilizando las escalas Símbolo-Dígito $(\mathrm{n}=26)$, TMT-A $(\mathrm{n}=29)$ y MMSE (n = 34). 

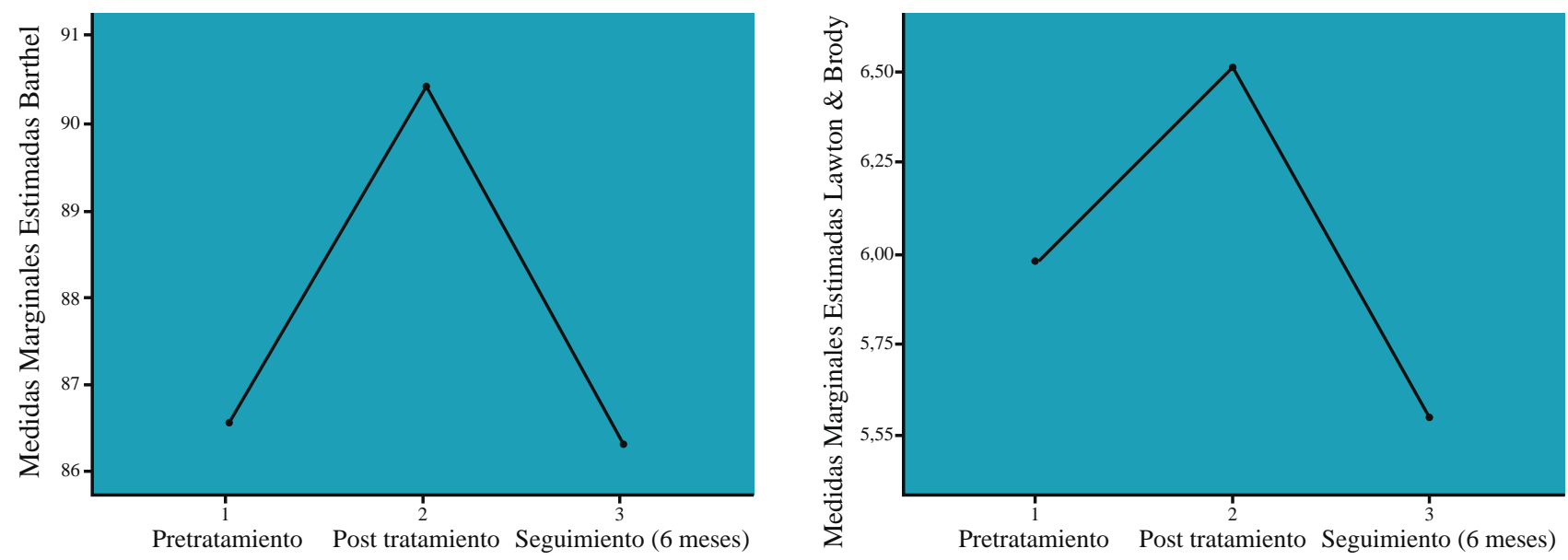

Figura 4. Media de las puntuaciones obtenidas en tres medidas en PA en la Escala de Lawton y Brody, y la Prueba de Barthel.

\section{Evaluación del efecto del programa mediante grupos focales}

A continuación se presentan los hallazgos obtenidos a partir del análisis de contenido de los cuatro grupos focales realizados con pacientes y los cuatro con cuidadores. Previo consenso entre investigadoras, se contabilizó la frecuencia de ocurrencia de citas, es decir el número de veces que ocurrió una expresión referida a cambio positivo en las diferentes categorías para cada variable, y se encontró que las categorías que reportaron mayor percepción de cambio fueron: memoria episódica, motivación, atención, vitalidad y agilidad (véase Tabla 3).

Tabla 3

Frecuencia de citación por categorías identificadas en grupos focales

\begin{tabular}{lll}
\hline Cambios en variables & $\begin{array}{l}\text { Categorías } \\
\text { identificadas }\end{array}$ & $\begin{array}{l}\text { Frecuencia } \\
\text { de citación }\end{array}$ \\
\hline $\begin{array}{l}\text { Cambios en calidad de } \\
\text { vida }\end{array}$ & Agilidad & 12 \\
& $\begin{array}{l}\text { Interacción } \\
\text { Autonomía }\end{array}$ & 9 \\
& Sueño & 4 \\
& Respiración & 3 \\
& Dolor & 2 \\
Cambios en variables & Motivación & 23 \\
afectivas & Vitalidad & 16 \\
& Seguridad & 7 \\
Cambios en variables & Memoria & 32 \\
cognitivas & episódica & 19 \\
& Atención & Memoria pro- \\
& cedimental & 9 \\
Cambios en variables & Otras activi- & 8 \\
& dades & \\
funcionales & Actividades & 6 \\
& instrumentales & \\
\hline
\end{tabular}

\section{DISCUSIÓN}

Este trabajo fue realizado con el propósito de aportar evidencia útil para ofrecer alternativas de manejo a los pacientes diagnosticados con enfermedad de Alzheimer e, indirectamente, a sus familiares. Su objetivo fue establecer el efecto de un programa de hatha yoga sobre la calidad de vida, la depresión, la atención, la memoria, el funcionamiento cognitivo global y la independencia en actividades funcionales básicas e instrumentales en adultos mayores de Bogotá con este diagnóstico.

Los resultados de la prueba inferencial de análisis de varianza no muestran evidencia estadísticamente significativa para ninguna de las mediciones cuantitativas que se tomaron a los pacientes entre el pre-tratamiento, el post-tratamiento y el seguimiento a los seis meses de finalizada la intervención; no obstante, se observó una convergencia en las tendencias que presentan las medidas de las diferentes variables. Esto permitiría sugerir una posible mejoría como efecto del tratamiento que en términos clínicos podría tener un impacto positivo para la calidad de vida de los pacientes; aunque es posible que dichas tendencias sean solamente efecto de la percepción subjetiva de los participantes. La falta de un grupo control limita el alcance de tales inferencias.

Las tendencias enunciadas son consistentes con la información aportada por los grupos focales que constituyen una fuente complementaria de información para detectar los cambios percibidos a partir de la aplicación del programa de intervención. A pesar del deterioro cognitivo que presentaban los pacientes (demencia leve-moderada), sus reportes sobre el efecto del programa fueron consistentes con las percepciones reportadas por sus cuidadores, aspecto que se considera como importante para comprender lo que significa vivir con demencia y que implica tener en cuenta 
la experiencia directa de los pacientes (Fromholt, Telenius, Engedal \& Bergland, 2015).

La calidad de vida fue evaluada mediante dos instrumentos (el ADQRL y el CV), la media de las puntuaciones obtenidas con ambas pruebas tuvo una tendencia de convergencia positiva durante el programa, aunque, luego de su finalización, una tendencia de convergencia negativa. Este patrón se repite con las puntuaciones de la variable “depresión”, medida con el cuestionario de depresión Yesavage; sin embargo, la variación en estas puntuaciones no implica cambios en el estado de ánimo. Además, el patrón mencionado se repite en las puntuaciones con las variables de independencia en las actividades de la vida cotidiana medida con la Escala de Lawton y Brody, y la Prueba de Barthel; y ocurre lo mismo con las variables de atención y memoria, medidas por la sub escala Dígito-Símbolo del WAIS-III y con la Prueba de Trazado (Trail Making Test Forma A), igual que cuando se evaluó el funcionamiento cognitivo global con el MMSE.

Lo anterior permitiría sugerir, a pesar de la limitación que implica el no contar con grupo control, una atenuación del deterioro con una leve mejoría en las capacidades de los pacientes que repercute positivamente en el mantenimiento de calidad de vida, la atención, la memoria y la independencia en sus actividades de la vida diaria. Entre las causas posibles de esta mejoría concomitante al programa de yoga podría estar el aumento en la producción de endorfinas que favorece un estado de ánimo positivo y sentimientos de bienestar, así como el fortalecimiento de cualidades físicas como la fuerza, el equilibrio y la coordinación, las cuales permiten una mejor y mayor funcionalidad en las actividades diarias (Pérez \& Cancela-Carral, 2008). Sin embargo, el efecto de las endorfinas es de carácter transitorio (Maggouritsa et al., 2014), hallazgo consistente con los resultados del presente estudio.

Por otra parte, el mantenimiento de las facultades mencionadas también pudo deberse a la práctica repetida de las posturas de yoga dado que los resultados de las investigaciones sugieren que niveles más altos y constantes de actividad física están asociados con un incremento en el flujo sanguíneo cerebral y, por ende, a un menor riesgo de daño cognoscitivo (Chapman, Marshall, Strine, Anda \& Moore, 2006). Por otro lado, diversas investigaciones han mostrado que el sistema neuroanatómico subyacente y responsable de la memoria procedimental es independiente al de la memoria declarativa y que, por tanto, los pacientes con Alzheimer pueden adquirir habilidades motoras, perceptuales y cognitivas; de acuerdo con esto, la conservación de la memoria implícita y del aprendizaje procedimental puede ocurrir hasta las últimas etapas de la enfermedad(Peraita \& Moreno, 2006), situación que podría estar relacionada con la oportunidad de aprender una rutina de actividad física que implica la ejecución de destrezas motoras y que está implícito en el programa de yoga. Para favorecer la permanencia en la fase de seguimiento de los efectos positivos observados en la fase de intervención, sería pertinente que en estudios futuros los pacientes fuesen expuestos a sesiones breves de mantenimiento en la fase de post-intervención.

Las tendencias antes descritas son consistentes con la evidencia previa (Coelho et al., 2012; De Andrade et al., 2013; Fan \& Chen, 2011; Khoo et al., 2014; Winchester et al., 2013). No obstante, ninguno de estos estudios tomó medidas de seguimiento que permitieran dar respuesta a la pregunta sobre el mantenimiento del efecto logrado y evidenciado a través de mediciones post-tratamiento. Por otra parte, los resultados del estudio de Cramer et al. (2014) son consistentes con el patrón de resultados obtenidos en la medición de seguimiento del presente estudio, los cuales podrían equipararse con las de largo plazo de estos autores. Finalmente, los resultados reflejados en el estudio de Sharma, Robbins, Wagner y Colgrove (2015) con pacientes con enfermedad de Parkinson concuerdan con los hallazgos de este estudio en el sentido de considerar la percepción subjetiva de los participantes como indicador de la efectividad del yoga para el mantenimiento del nivel de actividad física en los pacientes.

El programa de hatha yoga objeto de evaluación estableció una rutina de actividad física dirigida de dos horas por semana, con la invitación permanente a los cuidadores para apoyar a los pacientes para realizar en casa una o dos sesiones más semanalmente, en atención a la recomendación de US Department of Health and Human Services (2008) de practicar ejercicio físico aeróbico mínimo 150 minutos por semana. Sin embargo, dicha sugerencia no fue tenida en cuenta y en general la actividad física de los pacientes se limitó a las dos sesiones desarrolladas en compañía del instructor. En este sentido, los resultados obtenidos en cuanto a la ausencia de significancia estadística mediante el ANOVA de medidas repetidas podría explicarse en razón a que la intensidad de la actividad física semanal requerida para lograr un impacto efectivo en las variables de estudio no fue suficiente para asegurar su mantenimiento a lo largo de los seis meses de seguimiento ulterior. Esto constituye una de las limitaciones del presente estudio.

A partir de la experiencia obtenida con este programa de intervención, se establecen algunos lineamientos que podrían ser considerados en futuras investigaciones y propuestas de intervención con actividad física para población mayor con diagnóstico de demencia. El hatha yoga, gracias a que combina el ejercicio físico de bajo impacto (exigencia de las posturas) con la respiración y la relajación, y dada su 
exigencia atencional, es una alternativa de intervención no farmacológica útil para esta población; y aunque en el presente estudio no se midieron variables físicas como equilibrio y flexibilidad, estas fueron evaluadas en el estudio piloto (Quintero et al., 2011) en el que se obtuvieron resultados positivos demostrados también por otros autores (Donohue et al., 2006; McCaffrey et al., 2014; Riley, 2004); de esto se concluye que dichas variables deberían evaluarse en estudios futuros.

Para lograr una mayor efectividad del programa sobre las variables de estudio, se requerirían ciertos ajustes en el estilo de vida de los pacientes con el apoyo de su cuidador para que mantenga una práctica estable de la actividad física que permita acercarse al cumplimiento del estándar de 150 minutos semanales y tener un impacto favorable más duradero en la calidad de vida del paciente e indirectamente en la de su cuidador y de su familia. No obstante, esto puede resultar difícil en el contexto de esta población debido a las continuas complicaciones médicas que suelen presentar o a los cambios emocionales, actitud y ocupaciones del cuidador; aspectos que en este estudio podrían ser responsables de la atrición de la muestra de pacientes. Por otra parte, las intervenciones que promueven conductas y hábitos de vida saludables como comportamientos preventivos deben dirigirse también a población sana como, por ejemplo, a los cuidadores.

En este estudio, a diferencia del estudio piloto (Quintero et al, 2011), se incluyeron medidas cualitativas y métodos estadísticos mixtos que permitieron observar una tendencia convergente como efecto del programa, a pesar de las características de la muestra y de que la intensidad del ejercicio fue menor al estándar propuesto por la literatura. Es necesario tener en cuenta las limitaciones metodológicas del presente estudio para futuras investigaciones, como lo son la falta de un grupo control que permita evaluar el impacto del deterioro propio de la enfermedad en el grupo experimental y contar con una muestra mayor al inicio del estudio con el fin de disminuir el impacto de la atrición y conseguir una mejor potencia estadística en el análisis de datos.

Adicionalmente, para un nuevo estudio de evaluación de un programa de hatha yoga en pacientes con EA se debería incluir al menos tres sesiones por semana de una hora, bien sea presenciales o con el apoyo de las nuevas tecnologías virtuales que sirvan para incentivar, orientar y retroalimentar, incluso para ofrecer la posibilidad de interactuar con otras personas que realizan la misma actividad. Igualmente, con el fin de medir con mayor exactitud los cambios producidos por el programa, sería pertinente incluir medidas de variables físicas tales como equilibrio y flexibilidad, así como medir los niveles de cortisol de los que existe evidencia en la literatura de asociación a estados afectivos negativos.

\section{REFERENCIAS}

Ardila, A. Ostrosky-Solis, Roselli, M., \& Gómez, C. (2000). Age related cognitive decline during normal aging. The complex effect of education. Archives of Clinical Neuropsychology, 15, 6, 495-513.

Ardila, A. Roselli, M., \& Puente, A. E. (1994). Neuropsychological Evaluation the Spanish Speaker. New York: NY: Plenum Press.

Arlene, A. S.,Marieke, P., \& David, M. K. (2010). Effect of a 12-week yoga intervention on fear of falling and balance in older adults: a pilot study. Archives of Physical Medicine and Rehabilitation, 9, 576-583. doi: 10.1016/j. apmr.2009.12.018

Barnes, D.E., Mehling, W., Taffe, K., Flores, C., \& Chesney, M. (2013). Preventing loss of independence through exercise (PLIE'): an integrative exercise program for individuals with dementia. Neurology, 80 1, 353-362. doi: 10.1080/13607863.2014.935290

Chapman, D., Marshall, S., Strine, T., Anda, R., \& Moore, M. (2006). Dementia and Its Implications for Public Health. Preventing Chronic Disease, 3 (2), 1-13.

Chen, K. M., Chen, M. H., Hong, S. M., Chao, H. C., Lin, H. S., \& Li, C. H. (2008). Physical fitness of older adults in senior activity centers after 24-week silver yoga exercises. Journal of Clinical Nursing, 17, 2634-2646. doi: 10.1111/j.13652702.2008.02338.x

Cid-Ruzafa, J., \& Damián-Moreno, J. (1997). Valoración de la discapacidad física: el índice de Barthel. Revista Española de Epidemiología, 71, 127-137.

Coelho, F. G., Andrade, L. P., Pedroso, R. V., Santos-Galduroz, R., Gobbi, S., Costa, J. L. R., \& Gobbi, L. T. (2012). Multimodal exercise intervention improves frontal cognitive functions and gait in Alzheimer's disease: A controlled trial. Geriatrics \& Gerontology International, 13, 1, 198-203. doi: 10.1111/j.1447-0594.2012.00887.x

Cramer, H. et al. (2012). Yoga for breast cancer patient and survivors: a systematic review and meta-analysis. BMC Cancer, 18(12), 412. doi: 10.1186/1471-2407-12-412

Cramer, H., Lauche, R., \& Dobos, G. (2014). Characteristics of randomized controlled trials of yoga: a bibliometric analysis. BMC Complementary and Alternative Medicine, 14(1), 328. doi: 10.1186/1472-6882-14-328

Cramer, H., Lauche, R., Langhorst, J., \& Dobos, G. (2012). Effectiveness of Yoga for Menopausal Symptoms: A Systematic Review and Meta-Analysis of Randomized Controlled Trials, Depression and Anxiety, 00, 1-16. doi: $10.1155 / 2012 / 863905$

Creavin, S. T., Wisniewski, S., Noel-Storr, A. H., Trevelyan, C. M., Hampton, T., Rayment, D., \& Cullum, S. (2016). 
Mini-Mental State Examination (MMSE) for the detection of dementia in clinically unevaluated people aged 65 and over in community and primary care populations. Cochrane Database of Systematic Reviews. doi:10.1002/14651858. cd011145.pub2

Custodio, N., Gutierrez, C., \& García, A. (2007). Prevalencia de demencia en una comunidad urbana de Lima: un estudio puerta a puerta. Anales Facultad de Medicina, 69(4), 233238. doi: 10.15381/anales.v69i4.1110

Dayanim, S. (2009). The acute effects of a specialized movement program on the verbal abilities of patients with late-stage dementia. Alzheimer's Care Today, 10, 2, 93-98. doi: 10.1097/ACQ.0b013e3181a410ab

De Andrade, L. P., Gobbi, L. T. B. Coelho, F. G. M., Christofoletti, G., Costa, J. L. R., \& Stella, F. (2013). Benefits of multimodal exercise intervention for postural control and frontal cognitive functions in individuals with Alzheimer's disease: A controlled trial. Journal of the American Geriatrics Society, 61, 1919-1926. doi: 10.1111/jgs.12531

Donohue, B., Miller, A., Beisecker, M., Houser, D., Valdez, R., Tiller, S., \& Taymar, T. (2006). Effects of brief yoga exercises and motivational preparatory interventions in distance runners: results of a controlled trial. British Journal of Sport's Medicine, 40, 60-63.

Fan, J. T., \& Chen, K.-M. (2011). Using silver yoga exercises to promote physical and mental health of elders with dementia in long-term care facilities. International Psychogeriatrics/ IPA, 23(8), 1222-1230. doi: 10.1017/S1041610211000287

Ferri, C., Prince, M., Brayne, M., Brodaty, H., Fratiglioni, L., Ganguli, M., Hall, K., Hasegawa, K., Hendrie, H., Huang, Y., Jorm, A., Mathers, C., Mnezes, P. R., Rimmer, E., Scazufca, M., \& Alzheimer's Disease International. (2005). Global prevalence of dementia: a Delphi consensus study. The Lancet, 2112-2117. doi: 10.1016/S0140-6736(05)67889-0. Global

Fromholt, C., Telenius, E. W., Engedal, K., \& Bergland, A. (2015). Increased self-efficacy: the experience of high-intensity exercise of nursing home residents with dementia - a qualitative study. BMC Health Services Research, 15, 379-392. doi: 10.1186/s12913-015-1041-7

Gartside, S. E., Leitch, M. M., McQuade, R., \& Swarbrick, D. J. (2003). Flattening the glucocorticoid rhythm causes changes in hippocampal expression of messenger RNA scoding structural and functional proteins: implications for aging and depression. Neuropsychopharmacology, 28, 821-829. doi: $10.1038 /$ sj.npp.1300104

Graff-Radford, N.R. (2011). "Can Aerobic Exercise Protect against Dementia?", Alzheimer's Research and Therapy, 3(6), 1-6. doi: 10.1186/alzrt65

Gudlaugsson, J., Gudnason, V., Aspelund, T., Olafsdottir, A.S., Jonsson, P. V., Arngrimsson, S. A., \& Johannsson, E. (2013). Effects of exercise training and nutrition counseling on body composition and cardiometabolic factors in old individuals. European Geriatric Medicine, 4(6), 431-437. doi: 10.1016/j.eurger.2013.09.005

Henao-Arboleda, E., Muñoz, C. Aguirre-Acevedo, D. C., Lara, E., Pineda, D. A., \& Lopera, F. (2010). Datos normativos de pruebas neuropsicológicas en adultos mayores en una población colombiana. Revista Chilena de Neuropsicología, $5,3,214-226$

Hogervorst, E., Ribeiro, H. M., Molyneux, A., Budge, M., \& Smith, A. D. (2002). Plasma homocysteine levels, cerebrovascular risk Factors, and cerebral white matter changes (Leukoaraiosis) in patients with Alzheimer Disease. Archives of Neurology, 59, 787-793. doi: 10.1001/archneur.59.5.787.

Hophins, J. (2011). Enfermedad de Alzheimer y Calidad de Vida (ADRQL) - Instrumento de investigación, recuperado de http://www.hopkinsmedicine.org/psychiatry/specialty_ areas/geriatric_psychiatry/research/adrql.html

Howeison, D. B., \& Lezak, M. B. (2008). The neuropsychological evaluation. En S. C. Yudofsky \& R. E. Hales (Eds.) American Psychiatric Publishing Text Book of Neuropsychiatry and behavioral Neurosciences. (pp.215-244) Arlington, VA: American Psychiatric Publishing.

Jarcho, M. R., Slavich, G. M., Tylova-Stein, H., Wolkowitz, O. M., \& Burke, H. (2013). Dysregulated diurnal cortisol pattern is associated with glucocorticoid resistance in womenwithmajor depressive disorder. Biological Psychology,, 93, 150-158. doi: 10.1016/j.biopsycho.2013.01.018

Khoo, Y. J., Van Schaik, P., \& McKenna, J. (2014). The happy antics: holistic exercise for people with dementia. Bodywork and Movement Therapies, 18, 553-558. doi: 10.1016/j.jbmt.2014.02.008

Kjaer, T. W., Bertelsen, C., Piccini, P., Brooks, D., Alving., \& Lou, H. C. (2002). Increased dopamine tone during meditation-induced change of consciousness. Cognitive Brain Research, 13, 255-259. doi: 10.1016/S0926-6410(01)00106-9

Lawton, M. P., \& Brody, E. M. (1969). Assessment of older people: self-maintaining and instrumental activities of daily living. Gerontologist 9, 179-86

Li A.W. \& Goldsmith, C.A. (2012). The effects of yoga on anxiety and stress. Alternative Medicine Review, 17, 21-35. doi: $10.1016 /$ j.cct.2015.08.003

Liu, C.J., Latham, N.K. (2009). Progressive resistance strength training for improving physical function in older adults. Cochrane Database Syst Rev, 8 (3). doi: 10.1002/14651858

Maggouritsa, G., Kokaridas, D., Theodorakis, I., Patsiaouras, A., Mouzas, O., Dimitrakopoulos, S. \& Diggelidis, N. (2014). The effect of physical activity programme on improving mood profile of patients with schizophrenia. International Journal of Sport and Exercise Psychology, 12, 3, 1-12.

Mahoney, F.I. \& Barthel, D. (1965). Functional evaluation: The Barthel Index. Maryland State Medical Journal, 14, 56-61. 
Martínez de la Iglesia, J., Onís Vilches, M. C., Dueñas Herrero, R., Albert Colomer, C. A., Aguado Tabernés, C. \& Luque Luque, R. (2002). Versión española del cuestionario de Yesavage abreviado(GDS) para el despistaje de depresión en mayores de 65 años: adaptación y validación. Medifam, 12 620-630.

McCaffrey, R., Park, J., Newman, D., \& Hagen, D. (2014). The Effect of Chair Yoga in Older Adults with Moderate and Severe Alzheimer's Disease. Research in Gerontological Nursing, 7(4), 171-177. doi: 10.3928/19404921-20140218-01

Mittelman, M. S., Haley, W. E., Clay, O. J., \& Rith, D. L. (2006). Improving caregiver well-being delays nursing home placement of patients with Alzheimer disease. Neurology, 67, 1592-1599. doi: http://dx.doi.org/10.1212/01.wnl. 0000242727.81172 .91

Molero A. E., Pino-Ramírez, G., \& Maestre, G. E. (2007). High prevalence of dementia in a Caribbean population. Neuroepidemiology, 29, 107-12. doi:10.1159/000109824

Neuropathology Group of the Medical Research Council Cognitive Function and Aging Study. (2001). Pathological correlates of late-onset dementia in a multicentre, community-based population in England and Wales, The Lancet, 357(9251), 169-175. doi: 10.1016/S0140-6736(00)03589-3

Niedermeyer, E., \& Ghigo, J. (2011). Alzehimer dementia: an overview and promisisng new concept. American Journal of Electroneuro Diagnostic, 51, 82-91. doi: 10.1080/1086508X.2011.11079806

Oken, B. S., Zajdel, D., Kishiyama, S., Flegal, Dehen, C., Haas, M., Kraemer, D. F., Lawrence, J., \& Leyva, J. (2006). Randomized, controlled, six-month trial of yoga in health seniors: effects on cognition and quality of life. Alternative Therapies in Health Medicine, 12(1), 40-47.

Organización Mundial de la Salud. (2012). Guía de intervención mhGAP. Ginebra: Organización Mundial de la Salud.

Pages-Larraya, F., Grasso, L., \& Mari, G. (2004). Prevalencia de las demencias tipo Alzheimer, demencias vasculares y otras demencias en la República de Argentina. Revista Neurológica Argentina, 29, 148-153.

Peraita, H., \& Moreno, F. (2006). Análisis de categorías semánticas naturales y artificiales en una muestra de pacientes de Alzheimer. Psicothema, 3 (18), 492-500.

Pérez, C., \& Cancela-Carral, J. (2008). Benefits of physical exercise for older adults with Alzheimer's disease. Geriatric Nursing, 29 (6), 384-391. doi: 10.1016/j.gerinurse.2007.12.002

Pradilla, G., Vesga, B. E., Leon-Sarmiento F. E., Bautista L. E., Nuñez, L. C., Vesga E., Gamboa N. R., \& Grupo GENECO. (2002). Neuroepidemiology in the eastern region of Colombia. Revista de Neurología, 34, 1035-1043. doi:10.1590/ S1020-49892003000700005

Prince, M., Acosta, D., Chiu, H., Scazufica, M., \& Varghese, M. (2003). Dementia diagnosis in developing countries: a cross cultural validation study. The Lancet, 361, 909-17.
Quintero, E., Rodríguez, M. C., Guzmán, L., Llanos, O. L., \& Reyes, A. (2011). Estudio piloto: efectos de un programa de Hatha-yoga sobre variables psicológicas, funcionales y físicas, en pacientes con demencia tipo Alzheimer. Revista Psychologia, 5(2), 45-56. doi: 10.5007/1980-0037

Radak, Z., Hart, N., Sarga, L., Koltai, E., Atalay, M., Ohno, D., \& Boldogh, I. (2010). Exercise plays a preventive role against Alzheimer's disease. Journal of Alzheimer's disease, 20 (3), 777-783. doi: 10.3233/JAD-2010-091531

Ramos-Jiménez, A., Hernández-Torres, R. P., Wall-Medrano, A., Munoz-Daw, M. D. J., Torres-Duran, P. V., \& JuarezOropeza, M. A. (2009). Cardiovascular and metabolic effects of intensive Hatha yoga training in middle-aged and older women from northern Mexico. International Journal of Yoga, 2, 49-54. Tomado en febrero 26 de 2015 de http:// www.ncbi.nlm.nih.gov/pmc/articles/PMC2934576/. doi: 10.4103/0973-6131.60044

Riley, D. (2004). Hatha yoga and the treatment of Illnes. Alternative Therapies in Health and Medicine, 10(2), 20-26.

Rosselli, D., Ardila A., Pradilla G., Morillo, L., Bautista, L., Rey, O., Camacho, M., \& GENECO. (2000). The MiniMental State Examination as a selected diagnostic test for dementia: a Colombian population study. Revista de Neurología 30, 428-32.

Ruiz de S. C., Camargo L., Matallana D., Montañéz P., Reyes P., Zarco L., Pretelt, F., Nariño D., Coral J., Benito M., Quiroga F., \& Gálvez J. F. (2009). Una experiencia de 2600 valoraciones y 1918 casos de la clínica de Memoria de la Universidad Javeriana y el Hospital Universitario de San Ignacio durante doce años (1997 2009). Poster Congreso Colombiano de Neurología, Acta Neurológica Colombiana, 25, 170.

Sengupta, P. (2012). Health impacts of yoga and pranayama: a state of the art review. International Journal of Preventive Medicine, 3(7), 444-458.

Sharma, N.K., Robbins, K., Wagner, K., \& Colgrove, K. (2015). A randomized controlled pilot study of the therapeutic effects of yoga in people with Parkinson's disease. International Journal of Yoga, 8(1), 74-79. doi: 10.4103/09736131.146070

Skoog, I., Lernfelt, B., Landahl, S., Palmertz, B., Andreasson, L. A., Nilsson, L., Persoon, G., Odén, A., \& Svanborg, A. (1996). 15-year longitudinal study of blood pressure and dementia. The Lancet, 347: 1141-1145.

Smith, C., Hancock, H., Blake-Mortimer, J., \& Eckert, K. A. (2007). Randomized comparative trial of yoga and relaxation to reduce stress and anxiety. Complementary Therapies in Medicine, 15, 77-83. doi: 10.1016/j.ctim.2006.05.001

Smith, K.B., \& Pukall, C.F. (2009). An evidence-based review of yoga as a complementary intervention for patients with cancer. Psychooncoloy, 18, 465-475. doi: 10.1016/j. ctim.2006.05.001

Streeter, C. C., Gerbarg, P. L., Saper, R. B., Ciraulo, D. S., \& Brown, R. P. (2012). Effects of yoga on the autonomic 
nervous system, gamma-aminobutyric-acid, and allostasis in epilepsy, depression, and post-traumatic stress disorder. Medical Hypotheses, 78(5), 571-579. doi: 10.1016/j. mehy.2012.01.021

Tooley, G. A., Armstrong, S. M., Norman, T. R., \& Sali, A. (2000). Acute increases in night-time plasma melatonin levels following a period of meditation. Biological Psychology, 53, 69-78.

U.S. Department of Health and Human (2008) Physical Activity Guidelines for Americans: be active, healthy, and happy. Washington D.C.: Healther U.S. Gov.

Vadiraja, H. S., Raghavendra, R. M., Nagarathna, R., Nagendra, H. R., Rekha, M., Vanitha, N., Gopinath, K., Sirnath, B. S., Vishweshwara, M., Madhavi, Y. S., Ajaikumar, B. S., Rames, B. S., Nalini, R., \& Kumar, V. (2009). Effects of a yoga program on cortisol rhythm and mood states in early breast cancer patients undergoing adjuvant radiotherapy: a randomized controlled trial. Integrative Cancer Therapies, 8, 37-46. doi: 10.1177/1534735409331456
Vancampfort, D., Vansteelandt, K., Scheewe, T., Probst, M., Knapen, J., De Herdt, A., \& De Hert, M. (2012). Yoga in schizophrenia: a systematic review of randomised controlled trials. Acta Psychiatrica Scandinavica, 126, 12-20. doi: 10.1111/j.1600-0447.2012.01865

Wechsler, D. (1997). WAIS-III: Escala de Inteligência Wechsler para adultos - Manual David Wechsler. (3ª.ed.).

Winchester, J., Dick, M. B., Gillen, D., Reed, B., Miller, B., Tinklenberg, J., \& Cotman, C. W. (2013). Walking stabilizes cognitive functioning in Alzheimer's disease (ad) across one year. Archives of Gerontology and Geriatrics, 96-193. doi: 10.1016/j.archger.2012.06.016

Yu, F., \& Kolanowski, A. (2009). Facilitating aerobic exercise training in older adults with Alzheimer's disease. Geriatric Nursing, 30 (4), 250-259. doi: 10.1016/j.gerinurse.2008.11.001 